\title{
Lidil
}

Revue de linguistique et de didactique des langues

$63 \mid 2021$

Littératie numérique et didactique des langues et des cultures

\section{Écriture et lecture de chroniques en ligne : développer dans les interactions entre pairs ses compétences de littératie numérique}

Developing Digital Literacy Skills through Peer Interactions: The Case of Online

Communities of Writers and Readers of Urban Stories

Violaine Bigot et Nadja Maillard-De La Corte Gomez

\section{(2) OpenEdition}

Journals

Édition électronique

URL : http://journals.openedition.org/lidil/9239

DOI : 10.4000/lidil.9239

ISSN : 1960-6052

Éditeur

UGA Éditions/Université Grenoble Alpes

Édition imprimée

ISBN : 978-2-37747-283-3

ISSN : $1146-6480$

Référence électronique

Violaine Bigot et Nadja Maillard-De La Corte Gomez, «Écriture et lecture de chroniques en ligne

développer dans les interactions entre pairs ses compétences de littératie numérique », Lidil [En ligne],

63 | 2021, mis en ligne le 30 avril 2021, consulté le 28 avril 2021. URL : http://journals.openedition.org/ lidil/9239; DOl : https://doi.org/10.4000/lidil.9239

Ce document a été généré automatiquement le 28 avril 2021.

(c) Lidil 


\section{Écriture et lecture de chroniques en ligne : développer dans les interactions entre pairs ses compétences de littératie numérique}

Developing Digital Literacy Skills through Peer Interactions: The Case of Online Communities of Writers and Readers of Urban Stories

Violaine Bigot et Nadja Maillard-De La Corte Gomez

\section{Introduction}

1 Les «chroniques » sont des récits publiés en épisodes, depuis les années 2010, sur les réseaux sociaux et plateformes d'écritures (principalement Facebook et Wattpad) par des jeunes, le plus souvent des filles, résidant dans des quartiers périphériques de grandes villes ${ }^{1}$. La vie quotidienne y occupe une large place, les scènes au lycée y sont donc fréquentes. Échanges de textos entre élèves pendant les cours, conflits avec le personnel éducatif: le lycée apparait comme un lieu de sociabilité amicale suscitant ennui ou rébellion. Dans les rares temps de classe évoqués, les savoirs ou leurs modes de transmission sont tournés en dérision. En revanche, les échanges entre chroniqueuses et lectrices ou entre lectrices témoignent d'un rapport aux savoirs et savoir-faire très différent: ceux-ci y sont partagés pour soutenir et accompagner lecture et écriture.

2 On peut dès lors se demander quelles sont les pratiques de littératie numérique que contribue à développer la participation aux activités de lecture et d'écriture collaborative déployée autour des chroniques. Et en quoi le contexte numérique des 
échanges constitue-t-il un environnement favorisant le développement des compétences de littératie des chroniqueuses?

3 Nous commençons par présenter le corpus ${ }^{2}$ et les modalités de son exploration. Nous exposons ensuite le cadre théorique mobilisé et proposons une analyse du corpus où sont présentées d'une part les pratiques de littératie numérique que déploient chroniqueuses et lectrices dans ces échanges et d'autre part les modalités du développement collaboratif de ces compétences.

\section{Présentation du corpus}

4 Chaque page du réseau social Facebook de chronique ou chaque « histoire ${ }^{3}$ " publiée sur la plateforme d'écriture Wattpad peut être appréhendée comme un espace discursif à part entière associant les épisodes publiés et les commentaires postés en réaction à la chronique. Certaines caractéristiques (début et fin du récit, titre, url de la page...) lui confèrent une forme d'autonomie. D'autres (mention du genre «chronique » dans le titre ou les mots-clés, liens hypertextes vers d'autres espaces de chroniques...) le relient à un espace plus large que nous dénommons "espace réticulaire des chroniques ». Celui-ci comprend les pages de profil des chroniqueuses, celles que les fans consacrent à une chronique particulière ou à des sélections de chroniques. Cet espace réticulaire des chroniques constitue le terrain de recherche que nous explorons depuis cinq ans dans une démarche relevant de l'ethnographie du virtuel (Berry, 2012).

5 Cet article étudie les espaces discursifs de quatre chroniques, deux publiées sur Facebook (Petite Cendrillon amoureuse du prince du ghetto et Chronique d'une Zaïroise love d'un rebeu) et deux autres sur Wattpad (Chronique de Kadidia: Mariée de force à mon pire ennemi et Pour Aider Mon Père Je Me Suis Marié À Un Mafieu) $)^{4}$. Ces quatre chroniques sont très populaires et représentatives de différents sous-genres. Pour les chroniques Facebook (où les commentaires sont moins nombreux), 23 épisodes et les commentaires qu'ils ont suscités ont été retenus, pour les chroniques Wattpad, le corpus comprend cinq épisodes et leurs commentaires. Les 56 épisodes, les photos qui les accompagnent et les 40000 commentaires qu'ils ont suscités témoignent des pratiques de littératie numérique de la communauté et, à ce titre, nous renseignent sur la socialisation langagière numérique de ses membres. Nous avons privilégié l'étude des commentaires ${ }^{5}$ thématisant les savoirs et savoir-faire en jeu dans la lecture-écriture des chroniques et leur partage. Par ailleurs, trois pages de conseils sur l'écriture de chroniques, rédigées par Bbnala, Rosxnte et La Djazayriia complètent ce corpus.

\section{Cadrage théorique : participation aux espaces discursifs des chroniques et développement des pratiques de littératie}

6 Écriture et lecture en ligne de chroniques sont d'abord appréhendées ici comme des «pratiques ordinaires » de littératie (Chiss \& Marquillo-Larruy, 1998), relevant à la fois des pratiques de multilittératie et des pratiques de littératie numérique. Nous présentons ensuite l'intérêt des notions de participation et de socialisation langagière 
pour étudier le développement de ces pratiques au sein de ces communautés langagières en ligne.

\subsection{Multilittératie et littératie numérique}

7 Comme le soulignent les travaux du New London Group (Cope \& Kalantzis, 2009, p. 166), les pratiques de multilittératie relèvent du «multilingual » et du « multimodal ». Dans la plupart des récits se déploient des contacts entre langues et variétés de langue, donnant lieu à des commentaires abondants de lectrices. Certaines formes d'hétérogénéité montrée constituent une des caractéristiques stylistiques du genre (Bigot \& Maillard-De La Corte Gomez, 2020). La juxtaposition de variétés stylistiques très différentes contribue également à inscrire les chroniques dans le champ des new litteracies et incite à les aborder dans une perspective affranchie des logiques de standardisation et du prisme mononormatif prévalant parfois dans les études linguistiques (y compris sociolinguistiques) de l'écrit (Lillis \& McKinney, 2013). La multimodalité des chroniques en constitue une autre caractéristique: les chroniqueuses et leurs lectrices développent des pratiques plurisémiotiques diverses qui relèvent de ce que Paveau qualifie de « technographisme ", où l'on retrouve à la fois des jeux d'«iconisation du texte» (2017, p. 309) et de «conversationnalisation de l'image » (2017, p. 307).

8 Les chroniques peuvent être envisagées comme des pratiques de littératie numérique et à ce titre nous mobilisons pour les étudier les trois dimensions (interconnectées) de ces pratiques distinguées par Ollivier et Projet e-lang (2018) (dimension technologique, dimension de construction du sens et dimension interactionnelle). On retrouve, dans ces pratiques, la culture dite du «remix» dont Thorne (2010) a montré en quoi elle constituait un cadre facilitant pour l'écriture en L2.

9 Mais elles peuvent aussi être envisagées comme une forme renouvelée de certaines pratiques de littératie pré-numériques. Leur étude s'inscrit ainsi dans la continuité des recherches sur les écritures extrascolaires qui s'intéressent à ce qu'enfants et adolescents « font de l'écriture, lorsque rien ne les y oblige » (Penloup, 2007, p. 19). Ces recherches ont révélé dès la fin du $\mathrm{xx}^{\mathrm{e}}$ siècle l'importance quantitative et la diversité des pratiques d'écriture ordinaires. L'ère des réseaux sociaux numériques a rendu ces écrits accessibles d'une part à un nombre démultiplié de lectrices susceptibles de se lancer à leur tour dans l'écriture et d'autre part à la communauté des chercheurs.

L'étude des pratiques de littératie liées aux chroniques s'inscrit aussi dans l'histoire de la lecture. Chartier a montré qu'au-delà de l'activité cognitive, la lecture est « mise en jeu du corps, inscription dans un espace, rapport à soi ou à l'autre ", les "manières de lire propres à chaque communauté de lecteurs » devant être "reconstruites » (1989, p. 1512). Ici, le fonctionnement propre des réseaux et plateformes sur lesquels se déploient les chroniques permet la multiplication des échanges entre lectrices (et parfois avec l'autrice) et le développement de pratiques d'étayage mutuel dans l'activité de lecture. L'activité intense ainsi déployée pour mieux lire ensemble contribue à une forme de porosité - voire de fusion - entre activités de lecture et d'écriture, caractéristique des pratiques de littératie numérique. Les pratiques facilitées par le Web 2.0 confirment donc qu'« il n'est pas de compréhension d'un écrit quel qu'il soit qui ne dépende des formes dans lesquelles il atteint son lecteur » (Chartier, 1989, p. 1513). 


\subsection{Des espaces numériques de socialisation langagière}

Les interactions entre autrices et lectrices dans l'espace discursif réticulaire des chroniques construisent des "communautés de pratiques", c'est-à-dire, pour reprendre la définition de Wenger et coll. (2002), « groups of people who share a concern, a set of problems, or a passion about a topic, and who deepen their knowledge and expertise in this area by interacting on an ongoing basis » (p. 4). Dans les études de Lave et Wenger (1991) qui ont contribué à fonder cette notion, les processus d'apprentissage sont regardés en termes de participation à des pratiques sociales au sein d'une communauté, et non comme l'internalisation de savoirs ou savoir-faire sociaux, rendue possible par l'étayage ou l'instruction d'un expert. Cette participation implique tout l'individu (et non seulement sa dimension cognitive) en tant que personne engagée dans des activités et relations sociales aux autres membres du groupe. L'intégration d'un nouveau participant implique un double processus de transformation: le nouveau venu se développe grâce à la communauté et la communauté qui lui fait une place s'enrichit de sa présence. Berry (2007) a montré le potentiel du cadrage de Lave et Wenger pour l'étude des pratiques amateurs en contexte numérique en étudiant des guildes de joueurs en ligne. Les dynamiques d'intégration progressive des joueurs dans les communautés permettent que se développent conjointement leur potentiel d'action dans le jeu et leur rôle dans le devenir de ces communautés.

Tout comme celles portant sur les apprentissages situés dans des communautés de pratique, les études sur la socialisation langagière ne se focalisent ni sur le locuteur expert comme pourvoyeur d'un input ni sur le locuteur moins expert comme " acquérant» (acquirer), mais sur leur action conjointe permettant de «structurer les connaissances, les émotions, l'activité sociale» (Ochs, 2000, p. 230). Ces études définissent en outre la socialisation langagière comme socialisation au langage et par le langage (Schiefflin \& Ochs, 1986) : ce double mouvement se retrouve dans la participation à l'espace discursif réticulaire des chroniques, où se joue le développement d'une forme d'agentivité par les pratiques de littératie numérique et aux pratiques de littératie numérique. Dans cet espace, lectrices et autrices illustrent ces dynamiques de socialisation entre pairs qui «mettent au défi et subvertissent l'habitus d'une communauté » (Sterponi \& Bhattacharya, 2012, p. 75).

\section{Pratiques de littératie en jeu dans l'espace discursif interactionnel des chroniques}

13 Comment les activités d'écriture et de lecture des chroniques (dans les 3 dimensions des littératies numériques identifiées par Ollivier \& Projet e-lang, 2018, cf. supra) contribuent-elles à favoriser le développement de l'agentivité, comprise ici dans le sens large de pouvoir d'agir, des lectrices et des autrices?

\subsection{Coopérations technologiques au service de l'accessibilité, de la lisibilité et de l'esthétique icono-textuelle}

14 L'univers des chroniques se déploie sur différents réseaux sociaux et plateformes d'écriture; chroniqueuses et lectrices doivent être à même d'évoluer au sein de cet 
univers technologique en mutation, d'en comprendre les règles. Cette "capacité à identifier des outils et des ressources numériques (appareil, logiciel, application...) et à savoir les utiliser » qu'Ollivier nomme littératie «technologique » (Ollivier \& Projet e-lang, 2018, p. 10) se trouve explicitement thématisée, lorsque les lectrices demandent ou échangent des conseils.

Il s'agit tout d'abord de savoir se repérer au sein de cet espace numérique réticulaire, notamment sur Facebook qui n'est pas à priori une plateforme d'écriture. "Ou faut il aller pr la lire la chronique?» demande par exemple une nouvelle lectrice de Cendrillon (P1) à celles qui sont socialisées dans cet espace depuis plus longtemps - ignorant visiblement que les épisodes sont rangés dans un «album » accessible dans la rubrique « photo ».

De même, il est d'usage de demander « la suite » après la publication d'un épisode, pour marquer son intérêt et encourager la chroniqueuse à poursuivre: dans l'extrait 1 ci-après, une lectrice $(\mathrm{C} 1)$ demande "la suite » d'un épisode, alors qu'elle est déjà publiée depuis plus de quinze jours. La chroniqueuse (Zaïroise) lui offre alors un guidage technique pour accéder à la partie suivante puis l'accueille par un mot de bienvenue, après qu'une lectrice a souligné qu'elle était visiblement encore novice.

Extrait 1. - Zaïroise (Présentation)

$\mathrm{E} 1-\mathrm{C} 1 \mathrm{:j}$ viens juste de découvrir et je demande la suite

Zaïroise : Il suffit juste de cliquer sur la photo ya la suite :)

E1-C2 : Mdr elle vien juste de connaitre facebook jpense /.../ laiise lui le temps de

sabituer

Zaïroise : bienvenue a toi merci de suivre la chronique :)

Sur Facebook, les épisodes, publiés initialement sur le journal de la page, en tant que "légende» de photos, ne sont pas accessibles aux lectrices depuis leur téléphone. Celles qui lisent sur ordinateur prennent la peine de « copier-coller» les épisodes dans les commentaires pour les rendre accessibles. De nombreux messages sont adressés à ce propos soit à la communauté « kelkun peut metre le chronique comentaire je suis sur tel » (Zaïroise com-P1), soit à la chroniqueuse « tu peu le mettr en com avc le portable ont voi pa;((»(Cendrillon com-P1). Certaines chroniqueuses prennent les devants, guidant les lectrices dans l'espace des pages ( Ya la partie en comlentaire un peu plus haut 6062 :) » ou adoptent d'autres stratégies pour contourner les limitations des outils :

Extrait 2. - Zaïroise (1)

E2-C1 : T'as assure de mettre tes parties sur le mur la jsuis a fond!!!

Zaïroise : ha oui c'est vrai /.../ toi tu ne pouvais pas les voir avec ton phone j'espère que ca te va lol

Sur Wattpad, ce repérage concerne aussi l'espace réservé aux commentaires des lectrices en marge du texte : certaines lectrices alertent les « nouveaux qui commence a lire " sur cette «zone à risque » où des commentaires dévoilent parfois la suite du récit : « PARCE CE QU'ILS Y A DES VIELLES MEUFS QUI SPOIL ! ! ! ! (Kadidia - P1)6.

Les pages de conseils pour l'écriture de chronique, témoignent elles aussi des dimensions technologiques de ces pratiques de littératie. Rosxnte recommande non seulement de soigner l'orthographe, la ponctuation et les dialogues, mais elle insère une capture d'écran présentant une application gratuite "qui vous permets d'avoir plusieurs écritures» "pour ce soit beau». Bbnla souligne l'un des avantages de l'écriture numérique: "Ya de la place pour écrire c'est ça l'avantage de Wattpad par rapport à du papier » et poursuit en commençant un nouveau paragraphe : « Le texte 
doit être fluide, laisse les mots "respirer". » La Djazayriia évoque elle aussi la nécessité d'« aérer » le texte.

L'importance accordée à la "perception visuelle des textes » (Paveau, 2017, p. 308), caractéristique de l'ère des publications numériques, correspond aussi à une demande des lectrices. Une lectrice commente la première partie de Kadidia en conseillant « de faire des paragraphes moins long, ça donnera plus le goût à la lecture, comme ça là c'est un peu décourageant » et une autre de " raccourcir en /.../ séparant les paragraphes »: "c'est plus captivant tu vois?»

Outre l'accessibilité, la lisibilité et l'esthétique de la forme du texte, la place et le traitement de l'image font aussi l'objet de recommandations et de partages de ressources. Deux des trois pages de conseils étudiées mentionnent la question des photos ou photomontages qui donnent «envie de liker» (La Djazayriia). Rosxnte consacre un chapitre aux « couvertures » des chroniques, où elle donne entre autres ce conseil "si vous savez pas en faire demander à des filles qui en font sur wattpad", aussitôt mis en application par des lectrices qui s'échangent, dans les commentaires, des liens vers deux applications destinées à la réalisation de ces " covers ».

Dans l'écriture des chroniques, la dimension technologique concerne donc essentiellement les questions de mise en page et en image, soulignant l'attention portée à l'esthétique du texte (dimension de l'iconisation du texte) et à l'association de l'image au texte (dimension iconotextuelle). Cependant, le texte, élément central, garde une forme d'autonomie comme en attestent les republications des chroniques qui ne préservent pas les images initiales. De ce point de vue, la pratique des chroniques illustre une forme de résistance au pictorial turn et à «la domination de l'image sur le langage articulé» (Nachtergael, 2017, cité par Paveau, 2017, p. 308) qui caractériserait « la production intellectuelle et artistique » de notre époque.

\subsection{La construction du sens : collaborer pour comprendre et interpréter la chronique}

23 Dans l'espace discursif des chroniques, la compréhension et l'interprétation du texte comportent nécessairement une dimension collaborative. Les multiples codes en jeu dans toute lecture y sont coconstruits et sans cesse négociés.

\subsubsection{L'attention conjointe à la langue au service de la construction du sens des récits}

Dans les 3 pages de conseils, les conseils formels d'écriture occupent 5 des 26 chapitres : « l'écriture » et « le dialogue » (Rosxnte), « l'écriture » (La Djazayriia), « l'orthographe » et «les temps» (Bbnala). Les foyers d'attention sont variés : nécessité de traduire les dialogues en langue étrangère (La Djazayriia), orthographe et ponctuation (Bbnala et La Djazayriia), écriture des dialogues (Rosxnte et La Dajzayriia). La Djazayriia souligne aussi la nécessité de bien rendre le caractère multimodal de la communication entre les personnages et de distinguer graphiquement « les dialogues, les appels, les messages ».

Ces conseils visent à «toucher » le lecteur : «s'il n'y a pas de virgule de point et bah le texte sera fade, sans "émotion" » (Bbnala); à apporter un meilleur confort de lecture : "lire un texte bourré de faute, ça donne pas du tout envie de lire en plus sa saoule » 
(La Djazayriia), ou une meilleure intelligibilité : «c'est important de bien écrire pour une meilleure compréhension » (Bbnala).

Pour l'intelligibilité du texte, les lectrices peuvent également compter sur le soutien de la communauté qui développe une intense activité de coexplicitation du sens.

Prenons, à titre d'exemple, la partie 1 de Kadidia. Cinq segments textuels du récit font l'objet, dans les commentaires, d'une définition à la demande d'une lectrice. La question "Robe patineuse qui m'explique ? ? » trouve rapidement une réponse qui satisfait tout le monde, mais d'autres demandes déclenchent des séquences plus complexes. Ainsi, lorsque la chroniqueuse fait dire au frère de la narratrice : « je suis pas un PD pour que ma sœur sorte habilée comme ça », les propos du frère déclenchent 162 commentaires dont la question «Je peux savoir c'est quoi PD ?». Quatre lectrices collaborent à la construction d'une réponse expliquant le terme hors contexte (« c'est un gay " ou "un mec qui ce comporte comme une meuf genre un garçon comme une fille ») puis en contexte («Là il veut juste dire qu'c'est pas un imbécile pour la laisser sortir comme ça »). Une explication de «MDR » sollicitée, en anglais, par une lectrice qui se dit anglophone provoque aussi plusieurs échanges. L'onomatopée "tchuur " employée dans le récit ("Moi *tout doucement* tchhuur») suscite l'activité métalinguistique des lectrices: 95 commentaires dans la partie 1 et 60 dans la partie 119, dont des demandes d'explication, à l'instar de l'extrait 3 :

Extrait 3. - Kadidia (1)

E3-C1 : Ça veux dire quoi ça?

E3-C2: En gros cst un tshiip

E3-C1 : Ahhhhh jme disais bien /.../

Une partie des lectrices exprime son étonnement devant cette forme, voire remet en cause son existence ( ses “Tchiiip" ET NON “tchurrrr" *) »), d'autres s'engagent dans une réflexion collective où sont interrogés pêlemêle le sens de l'onomatopée, les options orthographiques pour la retranscrire, l'identité des locutrices qui l'utilisent... De même l'expression "fiontontonton » déclenche 109 commentaires où sont discutés le sens de l'expression («Niah Niah Niah quand quelqu'un t'enerve»), son aire d'usage (« ivoiriens», " angolais", " congolais», "Les maliens aussi en gros l'Afrique de l'ouest »), sa forme («fiontonton », « fiontontonton », «fiontonfionton ») et son sens.

On relève d'autres commentaires sur des segments linguistiques qui semblent très bien compris, mais dont la forme ou les usages sont discutés. Ainsi l'invocation «allah y rahmou ", qui accompagne dans Kadidia, la mention du décès de sa mère, est corrigée dans les commentaires en "allah y rahma " car " pour une femme ", " on accorde", mais elle fait aussi l'objet de débats car son usage pour des personnages fictifs (Kadidia est une chronique « fictive ») est jugé blasphématoire.

Ces séquences montrent comment la coopération entre lectrices permet qu'au-delà du sens dénoté de l'unité linguistique pointée, se discutent les connotations et implicites culturels qu'elle véhicule, les pratiques communicatives qui lui sont associées, mettant plus largement en jeu la compétence référentielle et/ou idéologique des lecteurs.

\subsubsection{Coconstruction du sens du récit : multimodalité et connaissance des codes du genre}

31 L'écriture et la lecture en ligne de ces récits fleuves postés en épisodes, sur une temporalité longue (Bigot \& Maillard-De La Corte Gomez, 2020) implique aussi, comme pour toute écriture en feuilleton, de (faire) comprendre les relations entre les 
personnages, de les resituer les uns par rapport aux autres. Pour ce faire, les pages de conseils partagent des astuces, et les lectrices coopèrent en cours de lecture.

En marge de la présentation des personnages de Pour aider mon père, trois lectrices

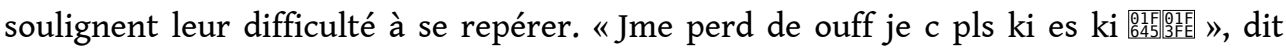
l'une d'entre elles. Deux autres ( $\mathrm{C} 1$ et $\mathrm{C} 4$ ) viennent partager leurs stratégies :

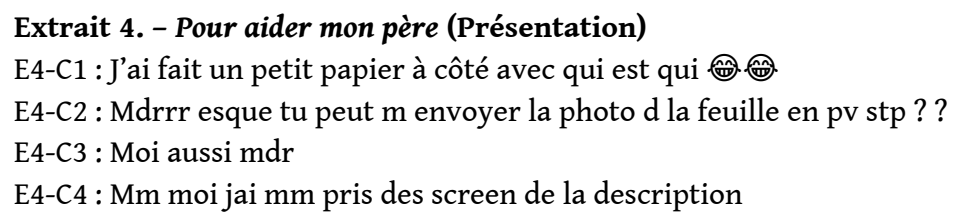

L'échange illustre la diversité des stratégies de construction de sens, caractérisées par leur dimension multimodale (notes sur « un petit papier ", « screen de la description ») et le principe de la mutualisation des ressources que permet l'espace des commentaires.

Dans l'extrait 5 , après une longue interruption de la chronique, une lectrice (C1) dresse la liste des personnages, rappelle leur identité, leur relation et sollicite des autres lectrices des informations complémentaires (ce que fait C2). La liste dressée n'étant visiblement pas exhaustive, $\mathrm{C} 1$ propose à ses paires une autre stratégie de lecture, prenant appui sur leur connaissance partagée du fonctionnement du genre : consulter l'incipit, traditionnellement dédié à la présentation des personnages.

\section{Extrait 5. - Kadidia (119)}

E5-C1: Kadidia: Personnage Principale Aïcha: Mère de Kadidia /.../ J'ai oublié comment s'appelle le père de Malick (dîtes le moi svp) /.../ Voila dîtes moi si j'ai oublié des personnages ou si je me suis trompée ! ! ! :- »/.../

E5-C2: Ya aussi Adama: Meilleur ami de Malik, devenu proche par la suite de Kadidia. Le LIEN de leur couple.

E5-C1 : Ah oui merci ! ! C'est vrai que j'ai oublié des personnes :-)

E5-C3: Tkt y'en a encore plus mdr : :-

E5-C1 : Bah dans ses cas la go voir la présentation des personnages :-)->

Sans doute forte d'expériences de lecture de ce type, Bbnala conseille d'ajouter aux chroniques un « album photo » dont le but est d'aider « les distraites ou celles qui lisent 27376 chroniques en même temps » à repérer les personnages. Cet album, précise-telle, doit donner les informations nécessaires à la compréhension de l'histoire, sans nuire au suspens, et s'appuyer si possible sur un support photo (" rapide et éfficase »).

Le maintien du suspens et plus largement de "l'originalité » des récits constitue un deuxième défi d'autant plus difficile à relever que les chroniques s'inscrivent dans un genre très stéréotypé. À travers leurs commentaires, certaines lectrices manifestent une "compétence rhétorique", reposant sur la «connaissance des genres", du "fonctionnement de certains types de textes ou de discours, la connaissance de catégories esthétiques et de "scénarios intertextuels" " (Rouxel, 2004, p. 21). Ainsi, dès la partie 1 de Pour aider mon père, un commentaire anticipe la suite prévisible de ce récit de mariage forcé : "On va te marié a un taré qui va te frapper mais plus vous serez ensemble plus vous vous aimerez. » Pour ne pas être accusée de "spoiler ", la lectrice poursuit : «Et je vous jure que j'ai pas lis la suite :-) », révélant en creux le caractère stéréotypé de ce genre de récit.

L'accès aux commentaires des lectrices contribue probablement à développer la sensibilité à cette question dans les pages de conseils d'écriture. Rosxnte met en garde contre le choix d'un titre qui révèlerait l'histoire et Bbnala explique dans un chapitre 
consacré à la question du suspens que celle qui parvient à relever le défi de «donner une claque au lecteur» "tout en gardant la logique» peut espérer lire des commentaires admiratifs du type « ooh nan je m'attendais pas à ça ».

\subsection{Socialisation entre pairs et développement de la littératie de l'interaction}

Définie comme la capacité "d'interagir et d'agir ensemble [...] en utilisant les technologies à disposition» (Ollivier \& Projet e-lang, 2018, p. 14), la littératie de l'interaction implique de savoir identifier le cadre participatif de l'échange (mail privé, forum, page publique, privée, etc.) et d'adapter son discours en conséquence. Toutes les pratiques technologiques et de construction de sens dont nous avons montré supra la dimension coconstruite révèlent les ressources nombreuses des chroniqueuses qui se saisissent des outils et les détournent pour interagir et partager des savoirs : espaces de «commentaire» devenant de vrais forums de discussion, «histoires» de Wattpad transformées en tutoriel d'écriture.

Les codes interactionnels contribuant à fédérer les communautés de lectrices ont été étudiés dans Bigot et Maillard-De La Corte Gomez (2017b). Nous y avons entre autres analysé des dynamiques subtiles de construction de connivence, fondées sur la mise en discours de différences et de points communs entre membres, et des stratégies de construction de la relation que nous avons qualifiées de pratiques de «bienveillance agrégative ». Mais les pratiques interactionnelles très consensuelles observées sur Facebook ont été perturbées lorsque le genre s'est développé sur Wattpad, plateforme d'écriture généraliste, où les lectrices qui commentent les chroniques ne maitrisent pas les codes d'échanges de la communauté. Les échanges relatifs à la maitrise de l'orthographe, analysés ci-après, illustrent bien ces « réglages interactionnels » avec les «nouvelles venues ».

Quasiment absente des échanges sur Facebook, la question de l'orthographe provoque sur Wattpad des recadrages fréquents. Ainsi, la phrase introductive de la partie 1 de Pour aider mon père : «Svp faite pas trop attention au faute d'orthographe » suscite une centaine de commentaires. Les lectrices réagissent majoritairement par des messages encourageant comme "tkt baby", "po grave", mais quelques-unes soulignent ironiquement les fautes qui émaillent la phrase d'excuse ou se plaignent du nombre de fautes d'orthographe dans les chroniques. Les lectrices socialisées depuis longtemps dans ces espaces leur rappellent le rapport libéré aux normes orthographiques qui y domine ( on écrit comme on veux en fait»), les attentes des lectrices (« on s'en blc avec les chroniques j'suis habituée $\mathrm{Mdr} »)$, revendiquent la spécificité de la publication numérique par rapport à la publication papier ("va lire des livres au lieu de nous clc ", " ceux qui sont pas satisfaits aller lire des dictionnaires pas des chroniques »). Enfin, la nature même de la relation construite par les commentaires normatifs est discutée. Une lectrice rappelle l'excuse qui accompagnait la remarque de la chroniqueuse sur son orthographe défaillante « Mais azy tve qw elle viens de s'excuser », une autre poste un simple « ok prof de français », ironisant sur le rapport de place et le rapport au savoir instauré par les postures normatives. 


\subsection{Modalités de développement de l'agentivité dans les pratiques de littératie en interaction}

41 Après avoir identifié les dimensions de la compétence de littératie numérique qui se développent dans les activités de lecture et d'écriture de chronique, nous étudions les dynamiques de partage des savoirs, des codes, des ressources et des stratégies ainsi que les modalités de collaboration qui soutiennent ces activités et contribuent au développement de l'agentivité des lectrices et des chroniqueuses.

\subsubsection{Apprendre pour lire / lire pour apprendre}

42 Nous avons vu supra que les postures normatives de lectrices critiquant l'orthographe d'une chroniqueuse suscitent des levées de bouclier de la part des autres lectrices. Mais pour de nombreux autres savoirs ou savoir-faire en jeu dans les activités de lecture et d'écriture, les partages de savoirs (notamment ceux issus des socialisations plurilingues et pluriculturelles familiales) sont nombreux, valorisés et encadrés par des rituels de politesse (cf. les « merci » et «stp » dans les extraits 3 et 5).

43 Les lectrices prennent plaisir à mettre en discours leurs apprentissages : ainsi dans Zaïroise, des commentaires thématisant les progrès jalonnent le récit : «le lingala j'fais des progrès " (partie 1), "Amy Poulloh hahaha à force de lire ta chronique je vais apprendre et comprendre le lingala » (partie 16) ou encore «J'ai appris kelke mots ausii "lingala" lol " (dernière partie). Cette mise en discours se manifeste aussi lorsque les lectrices réemploient des termes de la chronique qui ont fait préalablement l'objet de commentaires métalinguistiques. Nous avons ainsi étudié (Bigot \& Maillard-De La Corte Gomez, 2017a) la circulation de l'exclamatif «Hassilou » entre la chroniqueuse de Cendrillon et ses lectrices. De même, dans la partie 119 de Kadidia, une lectrice s'exclame : « Au lieu de tshiper je dis tchuuuuur maintenant », et témoigne ainsi de son appropriation de la forme de l'onomatopée si longuement discutée par les lectrices dans la partie 1.

44 Les demandes (ou offres) d'explication sont d'abord motivées par la volonté de mieux (aider à) comprendre la chronique. Les dynamiques peuvent être qualifiées de centripètes, recentrant les échanges vers le texte qu'il s'agit de comprendre ou d'interpréter, comme lorsque l'autrice répond à une lectrice qui exprime son empressement à lire la chronique : «Bonne lecture a toi et n'hésite pas a me parler au cas ou tu n'aurais pas compris qql choses Bisous ma belle $\bullet$ [41 dynamiques sont centrifuges, les échanges s'éloignant du texte pour aller vers d'autres savoirs. Le texte devient alors prétexte à enrichir son répertoire, comme le montre la demande d'explication d'une lectrice de Cendrillon des mots «mahlich /mashallah/ zehma/zahaf » utilisés dans le récit. La lectrice précise en effet: «Je vois ce que ça pourrait dire mais j'aime bien savoir... »

\subsubsection{Lire et être lue pour apprendre à écrire}

La manière dont les chroniqueuses développent leurs pratiques de littératie est notamment abordée dans les chroniques « réelles » lorsque le récit prend le temps d'un retour réflexif sur l'écriture et raconte sa propre genèse. La fonction cathartique de l'écriture est généralement mentionnée comme un puissant moteur; dans Cendrillon, partie 122, la chroniqueuse écrit : "J'ai ressenti le besoin d'écrire pour m'évacuer de 
toute la pression que j'avais sur le passé. » La découverte du genre est généralement à l'origine de la décision de se lancer dans une chronique. La lecture fait naitre l'écriture : "Alors j'ai décidé d'écrire cette chronique sur un coup de tête, vu que j'en lisais beaucoup [...] je me suis dis pourquoi ne pas en écrire une. » (Prologue de Kadidia)

Les chroniqueuses incitent par ailleurs parfois leurs lectrices à entrer dans l'écriture : celle de Cendrillon propose des " concours d'écriture ", et publie même, dans un album de sa page, une histoire dérivée de sa chronique et écrite par une lectrice (Dans la peau d'Hatem).

Une fois le processus d'écriture enclenché, comment les pratiques d'écriture des chroniqueuses se développent-elles?

Dans le prologue de Kadidia est souligné le rôle formateur de l'écriture elle-même: «Disclaimer : je sais que les premières parties sont mal écrites, en même temps c'était ma première fois il me fallait un temps d'adaptation. Une correction est prévu. » La qualité de l'écriture est donc destinée à s'améliorer au fil du temps.

Diverses formes de collaboration soutiennent par ailleurs les autrices dans leur activité d'écriture. Des livres sur Wattpad proposent des idées de trame narrative pour les chroniqueuses à court d'imagination. Kadidia s'associe, dès la partie 98, une correctrice-relectrice régulièrement remerciée ensuite : "Gros merci a dorkie_dee qui non seulement corrige les partie au plus vie mais qui galère avec moi à trouver les titres des parties». Des "admin» coaniment la page de Cendrillon et les lectrices l'enrichissent par leurs envois de "photo-montages». Dans la dernière partie de Pour aider mon père, intitulée : "Revelation de 2020 », la chroniqueuse révèle avoir raconté sa "vie à une autre fille qui a tout le talent ", son rôle s'étant limité à donner «quelques idées » et à relire « avant chaque publication ».

L'interaction avec les lectrices joue aussi un rôle central dans le développement de l'agentivité des chroniqueuses. La première partie de Kadidia se clôt par «Suite bientôt n'hésitez pas à donner votre avis... ». De nombreux commentaires postés pointent ensuite les qualités ou les défauts de la chronique, apportent conseils et critiques, rendant saillants des attendus du genre. Nous avions vu en 4.1 notamment les commentaires postés par des lectrices réclamant des paragraphes plus courts.

51 Mais ce type de remarque est toujours pris dans les dynamiques interactionnelles du Web 2.0: tout commentaire est susceptible d'être commenté à son tour, et toute prescription concernant l'écriture de chronique peut être négociée ou critiquée. Ainsi, les commentaires réclamant des paragraphes plus courts, sont aussitôt contrebalancés par les compliments d'une autre lectrice: «Elle sond grave long tes parti jaimme ttroop. »

52 À la première lecture, les pages de conseil sur l'écriture des chroniques relèvent d'un mode de construction des compétences plus «magistral », d'expert à non-expert. Néanmoins, les commentaires interrogent et discutent régulièrement les règles d'écriture ainsi formulées. À la fin de son chapitre $\mathrm{n}^{\circ} 1$ « L'organisation ", La Djazayriia synthétise ses conseils avec méthode: "Récapitulatif: Une idée -> Un titre -> un résumé personnel -> 10/15 parties prêtes avant la publication »; une lectrice/autrice lui oppose une autre stratégie :

Par "experience" tu dois savoir qu'un chapitre c'est énormément de travail donc il vaut mieux poser la un teaser, une introduction ou autre et voir les avis puis poster les chapitre un par un. Histoire d'avoir les critiques et avis pour s'améliorer au fur et a mesure des chapitres. 


\section{Conclusion}

Dans l'espace discursif des chroniques, les formes d'échanges contribuent à la socialisation des nouveaux venus qui peuvent compter sur le partage d'expérience des lecteurs ou auteurs socialisés depuis plus longtemps dans ces espaces. Mais la distinction entre membres socialisés depuis longtemps et nouveaux participants est ténue et les relations égalitaires vis-à-vis des savoirs sont fortement privilégiées. Dans les activités de colecture mais aussi de coécriture qui caractérisent ces espaces, la coopération est forte pour que se développent des pratiques technologiques, de construction du sens et d'interaction. Ces pratiques s'étayent mutuellement et contribuent au développement du pouvoir d'agir langagièrement dans des environnements numériques.

\section{BIBLIOGRAPHIE}

BERRY, Vincent. (2007). Les Guildes de joueurs dans l'univers de Dark Age of Camelot : apprentissages et transmissions de savoirs dans un monde virtuel. Revue française de pédagogie. Recherches en éducation, 160, 75-86. <https://doi.org/10.4000/rfp.738>.

BERRY, Vincent. (2012). Ethnographie sur Internet : rendre compte du « virtuel ». Les Sciences de l'éducation - Pour l'Ère nouvelle, 45(4), 35-58. <https://doi.org/10.3917/lsdle.454.0035>.

Bigot, Violaine \& MAILlARD-De LA CoRTE GomeZ, Nadja. (2017a). Processus de différenciation des pratiques langagières des jeunes et des adultes dans les chroniques facebook. Bulletin suisse de linguistique appliquée, numéro spécial, 117-128. <https://halshs.archives-ouvertes.fr/ halshs-01319904/document>.

Bigot, Violaine \& MAILlaRD-De La CORTE Gomez, Nadja. (2017b). « jkiff ! ! en plus moi osi chuis une $\mathrm{Z}$ ! »Construction de la connivence et reconnaissance de la différence dans le dialogue entre les chroniqueuses et leurs lectrices. Glottopol, 29, 111-135. <http://glottopol.univ-rouen.fr/ telecharger/numero_29/gpl29_05bigot_maillard.pdf>.

Bigot, Violaine \& MAILlaRd-De LA CORTE GomeZ, Nadja. (2020). Le bref et le long : les chroniques en ligne. Dans C. Meynard \& E. Vernadakis (dir.), Formes brèves. Au croisement des pratiques et des savoirs (p. 241-256). Presses universitaires de Rennes.

Bigot, Violaine, MAILlARD-De-LA CORTE GOMEZ, Nadja \& LAMBERT, Patricia. (2016). Les chroniques Facebook : étude exploratoire d'un genre d'écriture (très) populaire sur le Net. Dans Actes du $5^{e}$ Congrès mondial de linguistique française (CMLF) - SHS Web of Conferences (vol. 27). EDP SciencesWeb of Conferences. <https://doi.org/10.1051/shsconf/20162702003>.

Bigot, Violaine, MAILlARD-De LA CORTE GOMEZ, Nadja \& Noûs, Camille. (2020). Contacts de langue dans les espaces discursifs numériques des chroniques : la diversité linguistique en partage. Alsic, 23. <https://doi.org/10.4000/alsic.4772>. 
Chartier, Roger. (1989). Le Monde comme représentation. Annales. Histoire, Sciences Sociales, 44(6), 1505-1520. <https://doi.org/10.3406/ahess.1989.283667>.

CHISS, Jean-Louis \& MARQUILLÒ-LARRUY, Martine. (1998). Écriture et lecture : « literacy », pratiques ordinaires, pratiques lettrées. Dans F. Grossmann (dir.), Pratiques langagières et didactiques de l'écrit. Hommage à Michel Dabène (p. 75-84). IVEL-LIDILEM.

COPE, Bill \& KALANTZIS, Mary. (2009). “Multiliteracies”: New Literacies, New Learning. Pedagogies: An International Journal, 4(3), 164-195. <https://doi.org/10.1080/15544800903076044>.

LAVE, Jean \& WENGER, Étienne. (1991). Situated Learning: Legitimate Peripheral Participation.

Cambridge University Press.

LILLIS, Theresa \& McKInNEY, Carolyn. (2013). The Sociolinguistics of Writing in a Global Context: Objects, Lenses, Consequences. Journal of Sociolinguistics, 17(4), 415-439. <https://doi.org/10.1111/ josl.12046>.

NACHTERGAel, Magali. (2017). Le devenir-image de la littérature : peut-on parler de "neo-

littérature" ? Dans P. Mougin (dir.), La tentation littéraire de l'art contemporain (p. 291-304). Presses du réel.

OCHS, Elinor. (2000). Socialization. Journal of Linguistic Anthropology, 9(1-2), 230-233. <https:// doi.org/10.1525/jlin.1999.9.1-2.230>.

OllivieR, Christian \& PRojet E-LANG. (2018). Littératie numérique et approche socio-interactionnelle pour l'enseignement-apprentissage des langues. Éditions du Conseil de l'Europe. <www.ecml.at/Portals/ 1/5MTP/Ollivier/e-lang FR.pdf>.

PAVEAU, Marie-Anne. (2017). L'analyse du discours numérique : dictionnaire des formes et des pratiques. Hermann.

Penloup, Marie-Claude. (2007). Une écriture personnelle effervescente. Dans M.-C. Penloup (dir.), Les connaissances ignorées. Approche pluridisciplinaire de ce que savent les élèves (p. 17-44). INRP.

ROUXEL, Annie. (2004). Qu'entend-on par lecture littéraire ? Dans C. Tauveron (dir.), La lecture et la culture littéraires au cycle des approfondissements - « Les actes de la DESCO» (p. 19-30). Scéren / CRDP de l'académie de Versailles.

SCHIEFFELIN, Bambi \& OCHS, Elinor. (1986). Language Socialization. Dans B. Siegel (dir.), Annual Review of Anthropology, 15, 163-191.

STERPONI, Laura \& BHATTACHARYA, Usree. (2012). Dans les traces de Hymes et au-delà : les études de la socialisation langagière. Langage et société, 139(1), 67-82. <https://doi.org/10.3917/ls.139.0067>.

THORNE, Steven L. (2010). The "Intercultural Turn" and Language Learning in the Crucible of New Media. Dans S. Guth \& F. Helm, Telecollaboration 2.0 for Language and Intercultural Learning (p. 139-164). Peter Lang.

WENGER, Étienne, MCDERMOTT, Richard \& SNYDER, William M. (2002). Cultivating Communities of Practice: A Guide to Managing Knowledge. Harvard Business School Press.

\section{ANNEXES}


Annexe 1

Annexe 1 : présentation du corpus :

$1^{\text {tro }}$ partie du corpus : 56 épisodes + commentaires prélevés dans 4 chroniques.

\begin{tabular}{|c|c|c|c|c|c|c|}
\hline $\begin{array}{l}\text { Titre de la } \\
\text { chronique }\end{array}$ & $\begin{array}{l}\text { Publi- } \\
\text { cation } \\
\text { Faceb } \\
\text { ook/ } \\
\text { Wattp } \\
\text { ad }\end{array}$ & \begin{tabular}{|l|} 
Date de \\
publication ${ }^{1}$
\end{tabular} & $\begin{array}{l}\text { Nbre de } \\
\text { lectures } \\
\text { et } \\
\text { nombre } \\
\text { de votes }\end{array}$ & \begin{tabular}{|l} 
Nbre \\
total de \\
chapitres
\end{tabular} & chapitres étudiés & $\begin{array}{l}\text { Nbre total de } \\
\text { commentaires } \\
\text { postés pour } \\
\text { les parties } \\
\text { étudiées }\end{array}$ \\
\hline \begin{tabular}{l|} 
Pour Aider Mon \\
Père Je Me Suis \\
Marié A Un Mafieu \\
Par_deux_sheytans
\end{tabular} & WP & \begin{tabular}{|l|} 
Terminée \\
${ }^{* \prime}$ commentaire \\
$12-12-16$ \\
\end{tabular} & $\begin{array}{l}25,9 \mathrm{M} \\
1,7 \mathrm{M}\end{array}$ & 163 & $\begin{array}{l}\text { Présentation ; 1; } \\
80 ; 139 \text {, Fin }\end{array}$ & $16,3 \mathrm{~K}$ \\
\hline $\begin{array}{l}\text { Chronique de } \\
\text { Kadidia : Mariée de } \\
\text { force à mon pire } \\
\text { ennemi } \\
\text { ParMadamePogba }\end{array}$ & WP & 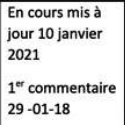 & $\begin{array}{l}18,7 \mathrm{M} \\
1,2 \mathrm{M}\end{array}$ & 134 & \begin{tabular}{|l|} 
Prologue, 1, 89, \\
119,120
\end{tabular} & 19,6 \\
\hline $\begin{array}{l}\text { Petite Cendrillon } \\
\text { amoureuse du } \\
\text { prince du Ghetto }\end{array}$ & FB & \begin{tabular}{|l|}
$11-10-2011$ \\
$23-03-2012$
\end{tabular} & 33670 & \begin{tabular}{|l|}
$125^{2}+$ \\
after chro
\end{tabular} & \begin{tabular}{|l|} 
Présentation, 1 à \\
20,80 et 123 \\
avant-première et \\
$123^{2}$
\end{tabular} & 672 \\
\hline $\begin{array}{l}\text { Chronique d'une } \\
\text { zaîroise love d'un } \\
\text { rebeu }\end{array}$ & $\mathrm{FB}$ & $\begin{array}{l}28-06-2011 \\
25-11-2011\end{array}$ & 17703 & 103 & \begin{tabular}{|l|} 
Présentation, 1 à \\
20,80 et 100 et \\
$100^{3}$
\end{tabular} & 1014 \\
\hline
\end{tabular}

1 Pour les publications Wattpad, ne sont visibles que les dates des commentaires, pour les

publications Facebook sont visibles les dates de publication des épisodes.

2 Les derniers épisodes de la chronique de Cendrillon sont numérotés respectivement 122, 122 bis

123 avant-première et 123.

3 Les deux dernières parties de la chronique sont toutes deux numérotées 100

$2^{\text {tme }}$ partie du corpus : 3 pages de conseils sur l'écriture des chroniques publiés sur Wattpad

\begin{tabular}{|l|l|l|l|}
\hline Nom du tutoriel & Nom de l'autrice & Nbre de chapitres & Nbre de lectures \\
\hline $\begin{array}{l}\text { Comment écrire } \\
\text { une chronique }\end{array}$ & la Djazayriia & 3 & $74 \mathrm{~K}$ \\
\hline $\begin{array}{l}10 \text { conseils pour } \\
\text { écrire une } \\
\text { chronique originale }\end{array}$ & Bbnala & 11 & $18,3 \mathrm{~K}$ \\
\hline $\begin{array}{l}\text { Ecrire une } \\
\text { chronique }\end{array}$ & Rosxnte & 11 & 3,6 \\
\hline
\end{tabular}

Annexe 2

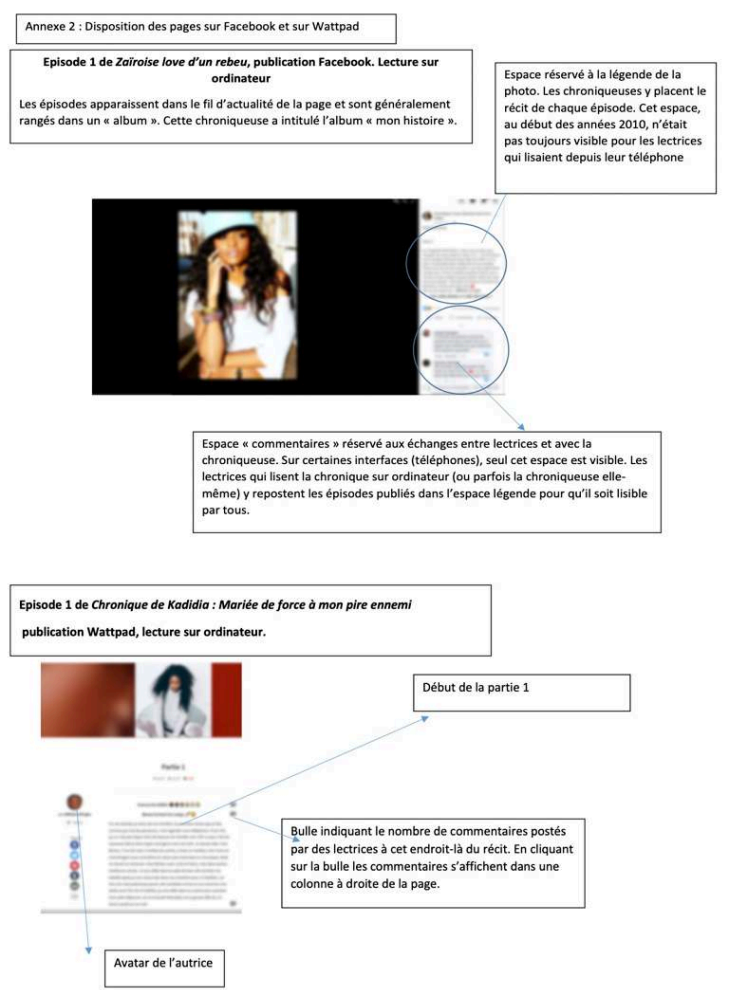




\section{NOTES}

1. Bigot et coll. (2016) pour une présentation plus exhaustive des caractéristiques du genre. Concernant le format des chroniques (longueur des récits en nombre de mots, rythme de publication), voir Bigot et Maillard-De La Corte Gomez (2020).

2. Dans l'annexe 1, un tableau présente le corpus ainsi réuni, et fournit un lien vers les chroniques évoquées dans l'article.

3. Toute page ouverte par Wattpad est catégorisée par la plateforme comme une « histoire».

4. Ci-après : Cendrillon, Zaïroise, Kadidia et Pour Aider Mon Père.

5. La comptabilisation du nombre de commentaires étudiés est délicate, un commentaire pouvant lui-même être l'objet de commentaires réactifs. La plupart des commentaires sont limités : un simple smiley ou un seul mot.

6. L'organisation de l'espace sur les pages Facebook et sur Wattpad est présentée dans l'annexe 2.

\section{RÉSUMÉS}

Les "chroniques", genre numérique natif du Web qui s'est déployé sur différents réseaux sociaux, notamment Facebook, occupent aujourd'hui une place conséquente sur la plateforme d'écriture Wattpad, mettant en lumière la place croissante occupée par les espaces numériques et les réseaux sociaux dans les pratiques de littératie des adolescents.

Cet article s'intéresse à la socialisation langagière s'opérant au sein des "communautés de pratiques» (Lave \& Wenger, 1991) réunies autour des pratiques d'écriture et de lecture des chroniques. Notre analyse interroge la diversité des savoirs et savoir-faire langagiers mis en discours dans ces communautés et s'intéresse aux modalités de transmission, partage, coconstruction de savoirs et savoir-faire pouvant y être observés. Nous nous intéressons aux interactions entre chroniqueuses et lectrices, et aux interactions entre lectrices, pour comprendre dans quelle mesure ces échanges étayent les activités d'écriture et de lecture numériques et, ce faisant, contribuent au développement des compétences de littératie numérique des membres.

Nous retenons pour cette étude un corpus réunissant quatre des chroniques parmi les plus populaires publiées sur Facebook et Wattpad, et trois « pages » de conseils sur les chroniques.

"Chroniques" are online fictional or autobiographical urban stories published in French on various social networks like Facebook. More recently they have developed on the French speaking part of writing platform Wattpad. This flourishing practice highlights the growing importance of digital spaces and social networks in adolescent literacy practices.

This article focuses on the language socialization that takes place within "communities of practice" (Lave \& Wenger, 1991) gathered around the practices of writing and reading chroniques. This article addresses the diversity of language knowledge commented on and developed by readers and writers and examines the methods of transmission, sharing, coconstruction of knowledge and know-how that can be observed in these communities. The research focusses on digital interactions between chronique writers and readers, and between readers' communities to understand to what extent these exchanges support digital writing and reading activities and, in so doing, contribute to the development of digital literacy skills of members. 
For this study, we have selected a corpus of four of the most popular "Chroniques" published on Facebook and Wattpad, and four pages providing advice to write chroniques on Wattpad.

INDEX

Keywords : urban romance, digital literacies, multiliteracies, language socialization, wild literacies

Mots-clés : chroniques, littératie numérique, multilittératie, socialisation langagière, littératie extrascolaire

\section{AUTEURS}

VIOLAINE BIGOT

Université Sorbonne Nouvelle, DILTEC EA 2288

violaine.bigot@sorgbonne-nouvelle.fr

NADJA MAILLARD-DE LA CORTE GOMEZ

Université d'Angers, CIRPaLL 7457

nadja.maillard@univ-angers.fr 